\title{
Dynamics of conjunctival impression cytologic changes after vitamin A supplementation
}

\author{
BY SWAPAN CHOWDHURY ${ }^{1}$, RAJESH KUMAR ${ }^{2}$, N. K. GANGULY ${ }^{1 *}$, LATA KUMAR ${ }^{3}$, \\ MINNI VERMA ${ }^{1}$ AND B. N. S. WALIA ${ }^{3}$ \\ Departments of ${ }^{1}$ Experimental Medicine and Biotechnology, ${ }^{2}$ Community Medicine and ${ }^{3}$ Paediatrics, \\ Postgraduate Institute of Medical Education and Research, Chandigarh-160012, India
}

(Received 26 July 1995 - Revised 8 October 1996 - Accepted 9 October 1996)

\begin{abstract}
To investigate the chronological changes in conjunctival epithelium after supplementation with a massive oral dose of vitamin A, conjunctival impression cytology (CIC) with transfer was carried out repeatedly among 200 children aged 6-120 months in a randomized, double-blind, placebo-controlled study in Chandigarh (India). Significant conversion to normal CIC started 71$80 \mathrm{~d}$ after vitamin A supplementation and by 101-110 d conversion had taken place in all children. Compared with the placebo group, plasma retinol concentration at $100 \mathrm{~d}$ post-supplement was found to be significantly higher in the vitamin $A$-supplemented group $P=0.04$. This study demonstrates that CIC responds to a massive oral dose of vitamin A 3-4 months after supplementation. These findings should guide future studies and evaluations in which CIC is used to assess response to vitamin $\mathbf{A}$ interventions.
\end{abstract}

Conjunctival impression cytology: Vitamin A: Retinol

Many developing countries have adopted oral supplementation with a massive dose of vitamin A administered every 6 months as a short-term strategy to prevent vitamin A deficiency. Long-term efficacy of a large oral dose of vitamin A in maintaining elevated plasma retinol concentrations is reported to vary widely from 2 to 42 weeks (West \& Sommer, 1987). Depending on the absorptive capacity, metabolic demand and reserves, functional availability of oral vitamin $A$ is also likely to vary in different populations. Plasma retinol concentration is not considered to be an accurate indicator of vitamin A status except at extremes of excess or deficiency (Olson, 1984).

Conjunctival impression cytology (CIC) is an alternative method for detection of vitamin A deficiency even in the pre-clinical stage (Amedee-Manesme et al. 1988; Natadisastra et al. 1988). However, to determine the change in vitamin A status, CIC needs to be done at an appropriate interval after supplementation with vitamin A. Natadisastra et al. (1987, 1988), Amedee-Manesme et al. (1988), Usha et al. (1990), and Carlier et al. (1992) repeated CIC at a fixed interval ranging from 2 weeks to 6 months following one or two massive doses of oral vitamin A. Information about the time interval at which CIC changes take place is not available.

The present study was conducted to determine the time required for conversion of abnormal conjunctival cytology to normal after vitamin A supplementation, and to study the plasma retinol concentration changes $100 \mathrm{~d}$ after a single, massive oral dose of vitamin A.

\footnotetext{
${ }^{*}$ For reprints.
} 


\section{SUBJECTS AND METHODS}

Two-hundred children aged 6-120 months who had no history of supplementation with a massive dose of vitamin $A$ within the previous 6 months and were not suffering from xerophthalmia, gross protein-energy malnutrition, or any other chronic disease were selected at random from a sampling frame of 1500 children from a slum community of Chandigarh, India. Informed consent from the parents and approval of the ethical committee of our Institute were obtained. In accordance with the protocol, during the study period, children were examined after every $15 \mathrm{~d}$ for detection of any sign or symptom of xerophthalmia, and morbidities including conjunctivitis. Children with xerophthalmia at any stage of the study were treated with two massive oral doses of vitamin $A$ at an interval of 1 week. Intake of large doses of vitamin A from other sources was enquired about and recorded. Children suffering from acute diseases or conjunctivitis were treated and exempted from sampling until their complete recovery.

CIC with transfer was used (Luzeau et al. 1988). A strip of cellulose-acetate paper (Celagram II, Shandon Southern Instruments Ltd, Camberley, Surrey) of approximately $3 \mathrm{~mm} \times 15 \mathrm{~mm}$ in size was applied to the bulbar conjunctiva for about $2-3 \mathrm{~s}$. This was taken off with gentle peeling. The impression collected on the paper strip was transferred immediately to a clean glass slide, applying light finger pressure. The slide with impression was placed in ethanol $(950 \mathrm{ml} / 1)$ for $15 \mathrm{~min}$, dried and kept in a staining solution containing a mixture $(2: 1, \mathrm{v} / \mathrm{v})$ of alcian blue $(2 \mathrm{~g} / 1 \mathrm{in} 50 \mathrm{ml} / 1$ acetic acid) and carbolfuchsin $(10 \mathrm{~g} / 1$ basic fuchsin in ethanol $(950 \mathrm{ml} / 1)$-phenol $(50 \mathrm{~g} / \mathrm{l}) ; 1: 1, \mathrm{v} / \mathrm{v})$ for $15 \mathrm{~min}$. After washing thoroughly in tap water, the slides were examined under a light microscope.

Sheets of small epithelial cells having a normal nucleus : cytoplasm ratio together with goblet cells and/or mucin spots, were regarded as being indicative of normal conjunctival epithelium, whereas enlarged epithelial cells of different sizes and shapes with small or pyknotic nuclei and absence of goblet cells and mucin spots were regarded as being indicative of abnormal conjunctival epithelium and indicative of vitamin A deficiency. A child with abnormal CIC in either eye was considered to have an abnormal CIC test. In case of failure of the CIC in one eye, the result from the other eye was used to categorize the child.

Plasma retinol concentration was estimated by HPLC (Thurnham et al. 1988). A sample of venous blood $(2 \mathrm{ml})$ was obtained through a 21 gauge needle with a syringe into a glass tube containing EDTA and wrapped with carbon paper. Samples were collected between 09.00 and 17.00 hours without fasting. Plasma was separated on the same day and stored at $-20^{\circ}$. Within 2 months of blood collection, retinol was extracted and analysed. In brief, $200 \mu \mathrm{l}$ plasma was mixed with $200 \mu 10 \mathrm{mM}$-sodium dodecyl sulfate. The sample was deproteinized with $400 \mu \mathrm{l}$ ethanol and finally extracted with $1000 \mu \mathrm{l} n$-heptane. After centrifugation at $2500 \mathrm{~g}$ for $10 \mathrm{~min}$, the supernatant fraction was transferred to another tube and dried under a gentle stream of $\mathrm{N}_{2}$ at $60^{\circ}$. The sample was then reconstituted in mobile phase (acetonitrile-methanol-chloroform, 47:47:6, by vol.) and injected into a $\mu$ Bondapak C18 column $(10 \mu \mathrm{m}, 3.9 \mathrm{~mm} \times 300 \mathrm{~mm})$, eluted at a flow rate of $1.8 \mathrm{ml} / \mathrm{min}$, and its retinol was monitored at $325 \mathrm{~nm}$ (Waters, Division of Milipore Corporation, Vienna, Austria).

After baseline sampling for CIC and plasma retinol, children were assigned at random in a double-blind fashion to receive either an oral dose of retinyl palmitate in $2 \mathrm{ml}$ arachis oil equivalent to $60000 \mu \mathrm{g}$ retinol or $200000 \mathrm{IU}$ for children $>12$ months (for children aged 6-12 months the dose was $1 \mathrm{ml}$, equivalent to $30000 \mu \mathrm{g}$ retinol or $100000 \mathrm{IU}$ ) or an equal volume of arachis oil as placebo. 
For repeat CIC all children of the vitamin $\mathrm{A}$ and placebo groups were divided at random into three sub-groups namely A1, A2, A3 for the vitamin A group and B1, B2, and B3 for the placebo group. Each sub-group contained thirty-three children. CIC samplings were done within $0-10,31-40,61-70$ and $91-100$ post-supplement days in sub-groups A1 and $B 1$, within 11-20, 41-50, 71-80 and 101-110 post-supplement days in sub-groups A2 and $B 2$ and within 21-30, 51-60, 81-90 and 111-120 post-supplement days in sub-groups A3 and B3. It was intended that children of each sub-group were tested for CIC five times (one baseline and four follow-up samples). For repeat plasma retinol concentration analysis on day 100, thirty children were selected at random from the vitamin A and placebo groups.

The McNemar test (Bourke et al. 1985) was used to compare the change in CIC status over time within each group. Student's $t$ test was used to compare plasma retinol concentrations between the different groups. Change in plasma retinol concentration over time within each group was tested by using paired $t$ tests. All $P$ values reported are twosided. A $P$ value of $<0.05$ was considered statistically significant.

\section{RESULTS}

Baseline sampling for CIC and plasma retinol concentration was not possible in two children due to non-cooperation, therefore they were not followed up. The baseline CIC status of eight children (four each in the vitamin A and placebo groups) could not be determined due to failure in taking the impression or bad staining of the samples, therefore their follow-up CIC samples, though available, could not be used for comparison.

Four follow-up CIC samples were obtained from 108 (54.5\%) children (forty-three in the vitamin A group and sixty-five in placebo group), three samples from seventy-five (37.9\%) (forty-seven in the vitamin A group and twenty-eight in the placebo group), two samples from ten (5.1\%) (six in the vitamin A group and four in the placebo group) and only one sample could be collected from five (2.5\%) children (three in the vitamin A group and two in the placebo group). Out of the total 1364 CIC specimens collected in the followup, $110(8 \%)$ could not be evaluated due to inadequate impression or bad staining. Of the 639 child examinations, fifteen $(2.2 \%)$ could not be evaluated because of failure to get a readable CIC specimen from at least one eye. Follow-up samples for plasma retinol concentration were obtained from twenty-nine (97\%) children of the vitamin A-group subsample and in seventeen (57\%) children of the placebo-group subsample on postsupplement day 100 .

The reasons for drop-out were: temporary migration from the study area $(17 \%)$, development of xerophthalmia ( $0.5 \%)$, intake of vitamin A from another source $(1 \%)$ and non-cooperation $(81.5 \%)$. The age, sex and baseline plasma retinol concentration were not significantly different among children who dropped out compared with children who were followed-up. The study population was particularly resistant to giving repeated blood samples. We had planned to study the CIC and plasma retinol concentration of all children at the end of the study, i.e. on post-supplement day 180. However, losses to follow-up on that day were great and only sixteen (27\% of the subgroups) plasma samples (twelve from the vitamin A group and four from the placebo group) and fifty-five (28\%) CIC samples (twenty-six in the vitamin A group and twenty-nine in the placebo group) could be obtained. The data for the 180th day are not presented since they are difficult to interpret due to significant losses as well as disparity of losses among the treatment groups.

Following vitamin A supplementation all children who were abnormal by $\mathrm{CIC}$ at baseline became CIC normal and no child who was CIC normal at baseline became 


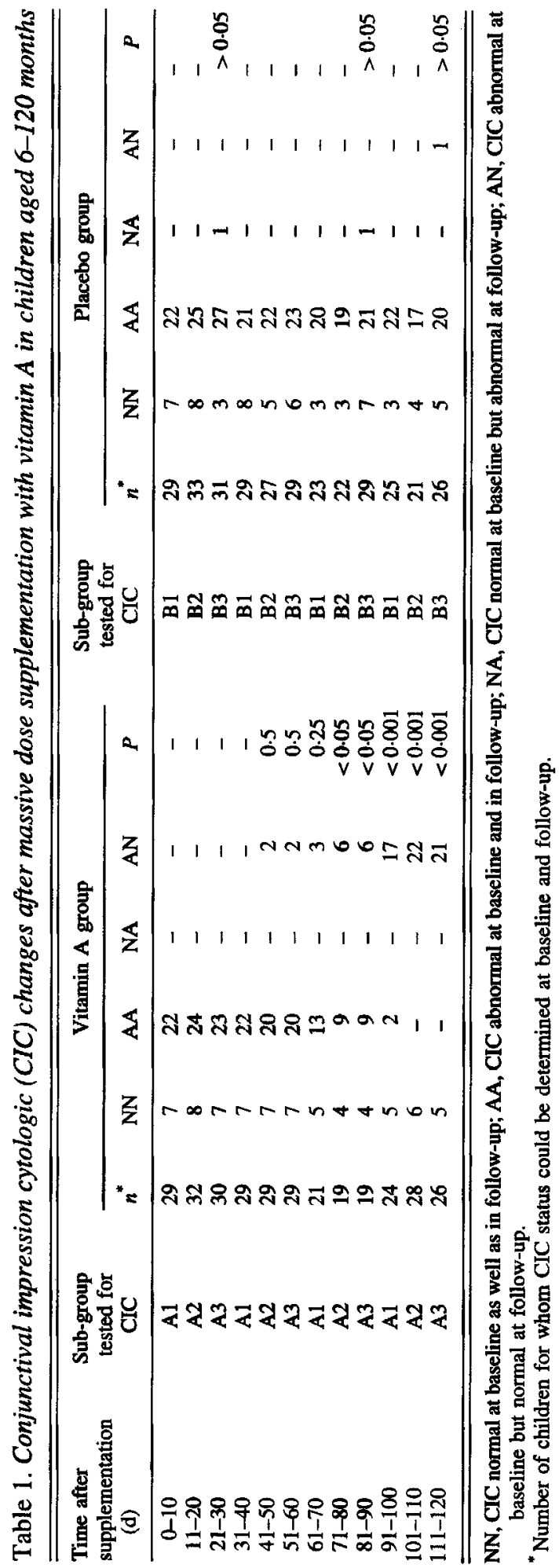




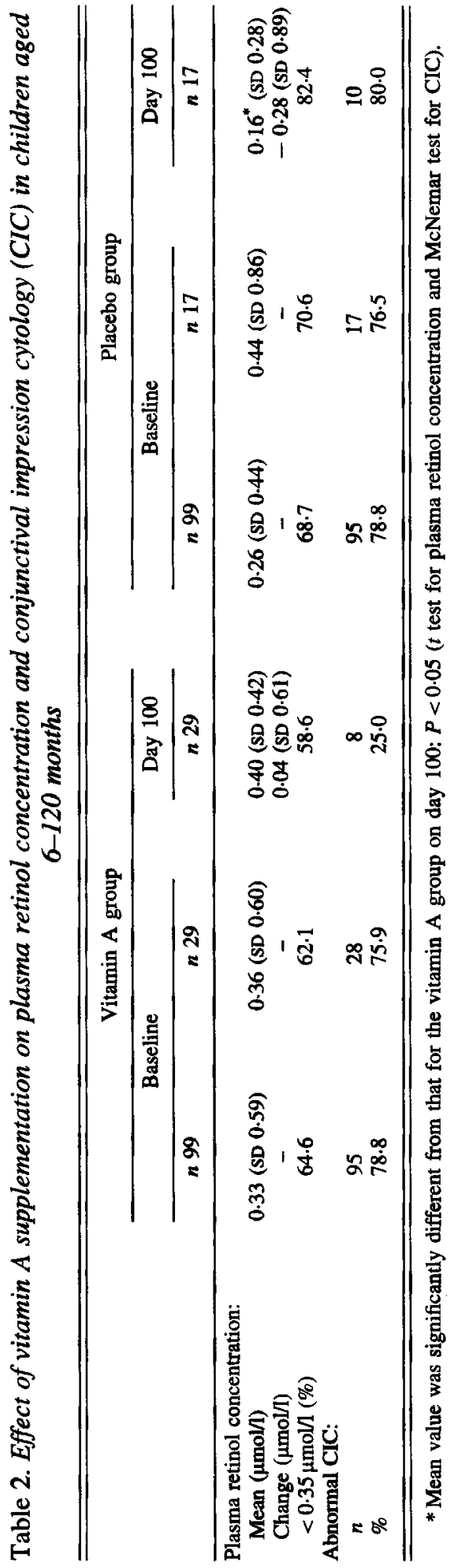


abnormal. Healing began 71-80 d after supplementation and was complete by $101-110 \mathrm{~d}$ (Table 1). In the placebo group one child who was abnormal by CIC at baseline became CIC normal, and two children who were normal became abnormal.

At baseline children with abnormal CIC had a mean plasma retinol concentration of $0.13 \mu \mathrm{mol} / \mathrm{h}$ compared with $1.09 \mu \mathrm{mol} / \mathrm{l}$ among those who had normal CIC. Mean plasma retinol concentration, proportion of children having plasma retinol concentration $<0.35 \mu \mathrm{mol} / \mathrm{l}$ and abnormal $\mathrm{CIC}$ at baseline and on day 100 after supplementation are presented in Table 2. Plasma retinol concentration in the vitamin A group was found to be significantly higher on day 100 compared with the placebo group $(P=0.04)$. The proportion of children with abnormal CIC declined from $79 \%$ to $25 \%$ in the vitamin A group $(P=0.01)$ whereas in the placebo group no significant decline was observed.

\section{DISCUSSION}

After vitamin A supplementation conversion of abnormal CIC to normal was observed in all children and none of them reverted to abnormal CIC within 4 months. Significant changes in CIC status were observed $70 \mathrm{~d}$ after supplementation (Table 1). Natadisastra et al. (1988) reported complete conversion of abnormal CIC to normal, 6 months after supplementation with vitamin A of non-xerophthalmic children with low plasma retinol concentrations. In another study, conversion of abnormal CIC to normal occurred in $95 \%$ of cases tested at 6 months after supplementation (Natadisastra et al. 1987). Carlier et al. (1992) reported that only $46 \%$ of subjects showing abnormal CIC had converted to normal 2 months after supplementation with vitamin A. Usha et al (1990) reported conversion of abnormal CIC to normal in sixteen children with persistent diarrhoea within $15-20 \mathrm{~d}$ after vitamin A supplementation. Children with acute deficiency of vitamin A may show conversion earlier than those with chronic deficiency owing to their comparatively exhausted reserves.

Plasma retinol concentrations of children with abnormal CIC were low in comparison with those of children with normal CIC at baseline in the present study. Similar observations were made by Carlier et al. (1992) in Senegal. Plasma retinol concentration was observed to be significantly higher in the present study after vitamin A supplementation on day 100 compared with that in children given the placebo (Table 2). However it is difficult to interpret the plasma retinol data since repeat blood samples could not be obtained from about half of the children due to non-cooperation in the placebo group. Drop-outs were less for CIC than for blood samples. People give a high value to blood since it is considered to be an essential element of life. Therefore, in developing countries CIC is more acceptable for use in field studies for assessment of vitamin A status.

The most significant finding of the present study is that CIC became normal in all children who had received the massive oral dose of vitamin A. This indicates that the oral dose of vitamin A was absorbed. Every child showed a positive response to vitamin A supplementation, but changes in the CIC did not start until $70 \mathrm{~d}$ after supplementation. The optimum response occurred about 3 months after supplementation. These findings should guide future studies and evaluations in which $\mathrm{CIC}$ is used to assess response to vitamin $\mathrm{A}$ interventions.

\section{REFERENCES}

Amedee-Manesme, O., Luzeau, R., Wittepen, J. R., Hanck, A. \& Sommer, A. (1988). Impression cytology detects subclinical vitamin A deficiency. American Journal of Clinical Nutrition 47, 875-888.

Bourke, G. J., Daly, L. E. \& McGiluray, J. (1985). Interpretation and Uses of Medical Statistics, 3rd ed., pp. 122-124. London: Blackwell Scientific Publications. 
Carlier, C., Etchepare, M., Ceccon, J. F., Mourey, M. S. \& Amedee-Manesme, O. (1992). Efficacy of massive oral doses of retinyl palmitate and mango (Mangifera indica L.) consumption to correct an existing vitamin A deficiency in Senegalese children. British Journal of Nutrition 68, 529-540.

Luzeau, R., Carlier, C., Ellrodt, A. \& Amedee-Manesme, O. (1988). Impression cytology with transfer. An easy method for detection of vitamin A deficiency. International Joumal for Vitamin and Nutrition Research $\mathbf{5 8}$, 166-170.

Natadisastra, G., Wittpenn, J. R., Muhilal, West, K., Mele, L. \& Sommer, A. (1988). Impression cytology: a practical index of vitamin A status. American Journal of Clinical Nutrition 48, 695-701.

Natadisastra, G., Wittpenn, J., West, K. P., Muhilal \& Sommer, A. (1987). Impression cytology for detection of vitamin A deficiency. Archives of Ophthalmology 105, 1224-1228.

Olson, J. A. (1984). Serum levels of vitamin A and carotenoids as reflectors of nutritional status. Journal of the National Cancer Institute 73, 1439-1444.

Thurnham, D. I., Smith, E. \& Flora, P. S. (1988). Concurrent liquid chromatographic assay of retinol, $\alpha$-tocopherol, $\beta$-carotene, $\alpha$-carotene, lycopene and $\beta$-cryptoxanthin in plasma with tocopherol acetate as internal standard. Clinical Chemistry 34, 377-381.

Usha, N., Sankarnarayanan, A., Walia, B. N. S. \& Ganguly, N. K. (1990). Early detection of vitamin A deficiency in children with persistent diarrhoea (letter). Lancet 335, 422.

West, K. P. \& Sommer, A. (1987). Periodic, Large Oral Doses of Vitamin A for the Prevention of Vitamin A Deficiency and Xerophthalmia: A Summary of Experiences. A Report of the International Vitamin A Consultative Group. Washington, DC: The Nutrition Foundation. 